\title{
THE INCIPIENT MODE OF STREAMERS IN A DIELECTRIC LIQUID AS A FUNCTION OF ELECTRIC FIELD
}

\author{
By: \\ M. Kim \\ S.D. Reedy \\ R.E. Hebner
}

15th IEEE International Conference on Dielectric Liquids (ICDL 2005), Coimbra, Portugal, June 26-July 1, 2005, pp. 71-74.

PR 400

Center for Electromechanics

The University of Texas at Austin

PRC, Mail Code R7000

Austin, TX 78712

(512) 471-4496 


\title{
The Incipient Mode of Streamers in a Dielectric Liquid as a Function of Electric Field
}

\author{
M. Kim, S. D. Reedy, and R. E. Hebner \\ University of Texas at Austin \\ Austin, Texas, USA
}

\begin{abstract}
Many previous studies of electric breakdowns in dielectric liquids in point-plane geometry have examined the relationships among the breakdown structure (or speed) and the electrode geometry (point radius, gap length) and/or voltage. This paper explores the hypothesis that the electric field, not the geometry per se, is the determining factor in the incipient streamer structure in dielectric liquids. This work extends earlier work and explores the extent to which the processes that determine initiation and growth are similar. This paper makes it explicit that geometry and voltage are indirect factors in the prebreakdown process, important only insofar as they determine the electric field in the liquid.
\end{abstract}

\section{INTRODUCTION}

Previous studies [1-4] of electric breakdown in dielectric liquids in positive-point point-plane geometry have shown that the initiation voltage for a prebreakdown streamer is a function of both tip radius and gap spacing. The initiation of electrical breakdown under these conditions in an insulating liquid is expected to be an electron-dominated process. Fundamentally, the force, F, on an electron of charge, e, is given by

$$
F=e E,
$$

where $E$ is the electric field. Thus, the energy deposited in the fluid is proportional to the kinetic energy the electron accumulates before colliding with a molecule, i.e. $e E \cdot \lambda$, where $\lambda$ is the path length over which the electron was accelerated. If the energy transferred in the collision between an electron and a molecule is less than the ionization energy for the molecule, the collision energy can be absorbed as heat, ultimately leading to localized boiling. For energy transfers greater than the ionization energy, a streamer can be initiated. As noted previously [5], the lower energy picture is consistent with the formation of the $1^{\text {st }}$ anode mode, while the $2^{\text {nd }}$ anode mode is consistent with more energetic collisions.

Because the electric field generically is a product of the applied voltage and a function appropriately representing the geometry, for the description above to be consistent with measured behavior, the changes in geometry and voltage must combine to yield a constant electric field. To test this hypothesis, the electric fields of previous works [3, 4] are analyzed with the boundary element method to generate more precise values than analytic solutions, which require an approximation of the geometry for a closed-form solution.
The electric field needed to produce a critical volume for streamer initiation is also shown. The analysis demonstrates that the previous works are consistent in the electric field.

\section{CRITICAL VOLUME}

For streamer initiation, a volume-time relationship must be a factor. In gaseous breakdown, it has been shown that, for given geometry and voltage, the probability $d P$ that a first critical avalanche will be created during time interval $d t[6]$ is given by

$$
d P=(1-P) \int_{\Gamma_{c}} \frac{d n_{e}}{d t} P_{0} d \Gamma d t
$$

where $d n_{e} / d t$ is the electron production rate, $P_{0}$ is the probability that electrons will remain active, and $\Gamma$ is the volume. The critical volume $\Gamma_{c}$ in gases is defined by two contour lines of constant electric field. The line furthest from the point electrode is the line at which an electron at that surface experiences a high enough electric field to initiate ionization upon collision with an atom or molecule. The contour nearer the electrode is the surface of maximum field strength at which an electron does not have adequate distance to produce an avalanche, because too few collisions are possible before impacting the anode. Given the higher density of liquids than gases, it is not clear a priori how well this concept can be applied to the initiation of streamers in liquids. This work provides some information on that topic.

The solution of (2) is given by

$$
P(t)=1-\exp \left(\int_{t_{0}}^{t_{s}} \int_{\Gamma_{c}} \frac{d n_{e}}{d t} P_{0} d \Gamma d t\right),
$$

where $t_{s}$ is the statistical time lag and $t_{0}$ is the time at which the streamer inception level is exceeded. The fundamental quantity required for calculating the statistical time lag is the rate of production of initiatory electrons. The production rate of effective electrons is given by

$$
R_{e}(t)=\int_{\Gamma_{c}(t)} \frac{d n_{e}}{d t} P_{0} d \Gamma
$$


Key factors for streamer initiation are growth of $\Gamma_{c}$ with applied voltage and $d n_{e} / d t$.

A different assumption has also been used in dielectric liquids with a high degree of success. Kupershtokh and colleagues [7, 8] have postulated that, while the streamer is propagating, the field in the vicinity of a streamer tip should be expressed as $E_{0}$ $+\delta$, where $E_{0}$ is the electric field strength necessary for breakdown and $\delta$ is a fluctuation in the electric field caused by changes in the local dielectric constant due to such factors as density fluctuations or molecular excitations. Using this assumption, they are able to develop a stochastic model that produces streamer growth patterns that are consistent with the growth patterns that have been observed in experiments.

There is no discrepancy between the field fluctuation assumption and the electro-optical observation that the field between the streamer and the electrode to which it is propagating can be modeled assuming the streamer is a conductor [9]. The experimental observations did not have sufficient precision to detect small, highly localized fluctuations in the electric field.

There is a discrepancy, however, between the field fluctuation model and the critical volume model that embeds the stochastic behavior in the production of electrons rather than in electric field variations [10]. While it is likely that the higher density of a liquid than a gas leads to variations in the local dielectric constant, there is not sufficient evidence to determine which stochastic process dominates. As shown in (3), the production of the necessary electrons to initiate a streamer is controlled by both the rate of free electron production and the electric field. They are very difficult variables to separate experimentally.

This work demonstrates that the critical volume model provides a framework in which different experimental results can be reconciled. It does not, however, show which fundamental process dominates under the conditions tested. That is still an unresolved question.

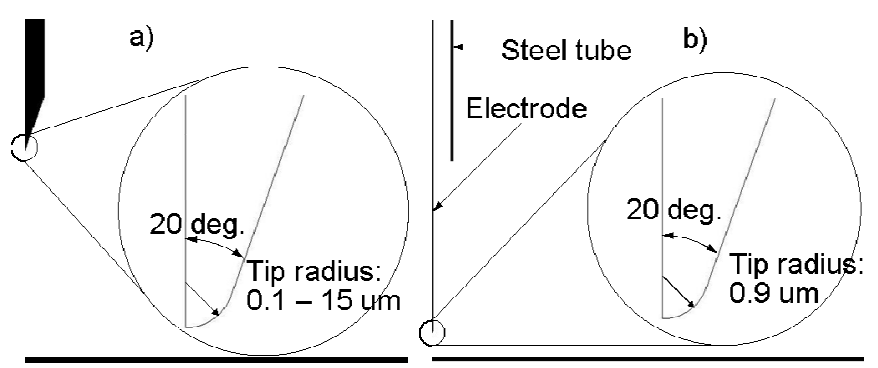

Fig.1. Analysis configurations of a) [3] and b) [4].

\section{RESULTS}

Theoretically, the initiation of a streamer should be determined by an electric field, not by a voltage. In both [2] and [3], the variations of initiation voltage with changes in geometry were reported. To obtain greater consistency, the electric field distributions with the geometries from $[3,4]$ were calculated assuming no variation in the azimuthal direction in cylindrical coordinates. The positive streamer case of [3] is analyzed to show its consistency with [4]. Tip radius is different in the two experiments. The configuration of this analysis is shown in Fig. 1 a). The analyzed tip radii range between $0.5 \mu \mathrm{m}$ and $15 \mu \mathrm{m}$, and the distance between the tip and the plate electrode is kept at $5 \mathrm{~mm}$. Fig. 2 shows that the results of this analysis are in good agreement with [3]. a)

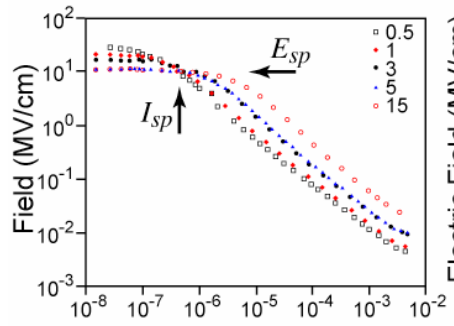

Distance from the needle tip $(\mathrm{m})$ b)

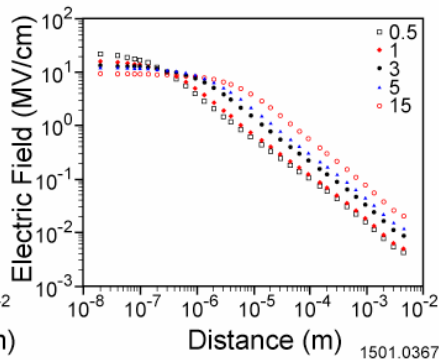

Fig.2. Electric field distribution from a) [3] and b) the analysis.

To clarify the relationship between the field distribution and tip radius at streamer initiation, a more precise analysis is needed of the field distribution near the tip. For a $0.5 \mu \mathrm{m}$ tip at a voltage of $9.3 \mathrm{kV}$, Fig. 3 shows the distance from the tip that a given percentage of the maximum field strength occurs for various tip radii. The sign of slope changes between $40 \%$ and $50 \%$. Consequently, there might be a distance having a constant electric field between $9.62 \mathrm{MV} / \mathrm{cm}(40 \%)$ and 12.03 $\mathrm{MV} / \mathrm{cm}(50 \%)$. The distance is between 0.2 and $0.4 \mu \mathrm{m}$. This suggests, in one dimension, that there might be a consistent field for initiation having a size scale in which the initiation can be detected optically. This led to a closer examination in two, or due to rotational symmetry, three dimensions.

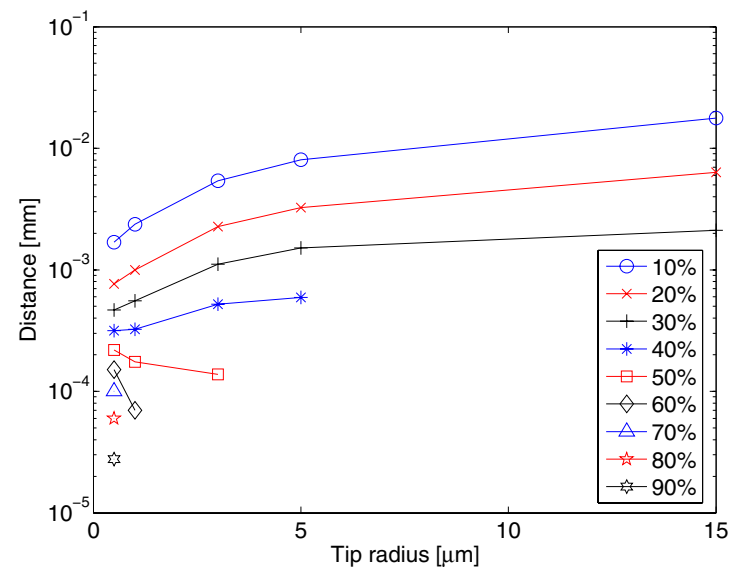

Fig.3. Distance with chosen electric fields. 
An additional confounding factor occurs due to the finite rise time of an applied voltage pulse. Fig. 4 shows how a presumed critical volume, the inner bound of the surface having constant electric field, changes shape with changes in field strength. In each of these four cases, the applied voltage and the geometry, including tip radius, are the same. The contours of constant fields are for $E_{a}>E_{b}>E_{c}>E_{d}$. This calculation suggests that if the voltage were raised on time scales comparable to the production rate of electrons or field fluctuation times, initiation of a streamer could occur at the tip, on the side of the shaft, or both. Fig. 5 shows streamers not only at the end of tips but also at the side of tips for both positive and negative streamers. The upper four frames show negative streamers and the lower ones, positive streamers. The streamers from the side are shown in both cases.

Using data like that from Fig. 3 and Fig. 4, the critical volume was calculated for a range of electric field strengths providing insight as to how a critical volume can change with tip radius. The critical volume increases if the electric field defining the critical volume is between $10 \%$ and $40 \%$ of the peak field, and decreases steeply if the field is between $50 \%$ than $60 \%$ of the peak. For an electric field of approximately $12 \mathrm{MV} / \mathrm{cm}$ $(50 \%)$, the critical volume is between $0.2 \mu \mathrm{m}^{3}$ and $0.5 \mu \mathrm{m}^{3}$.

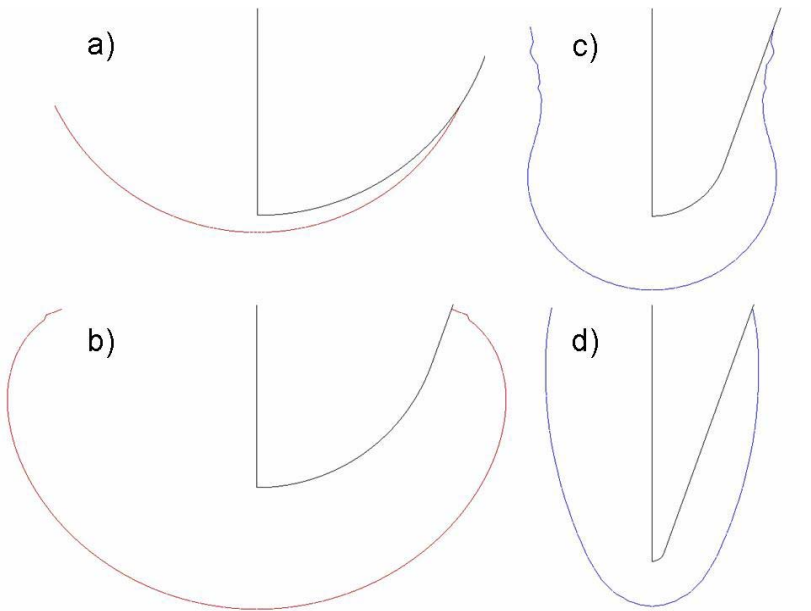

Fig.4. The shapes of electric field lines. a) The dish type, b) the apple type, c) the keyhole type and d) the ellipsoid type.

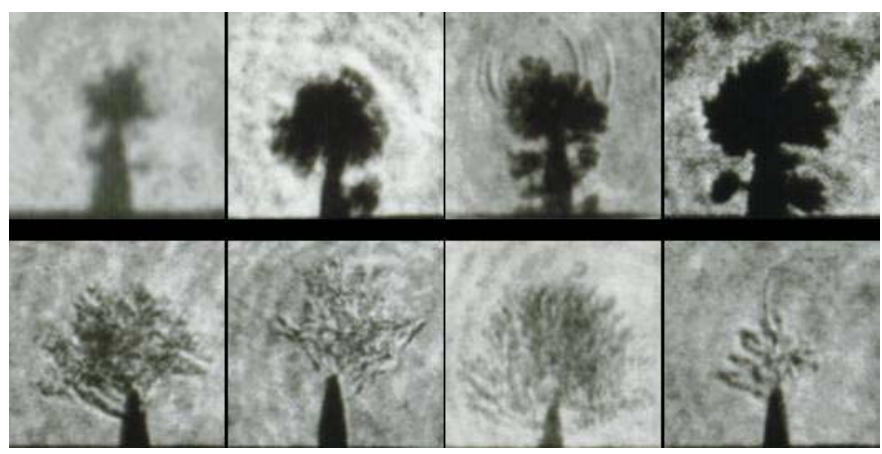

Fig.5. Negative streamers in the top row and positive streamers in the bottom row.

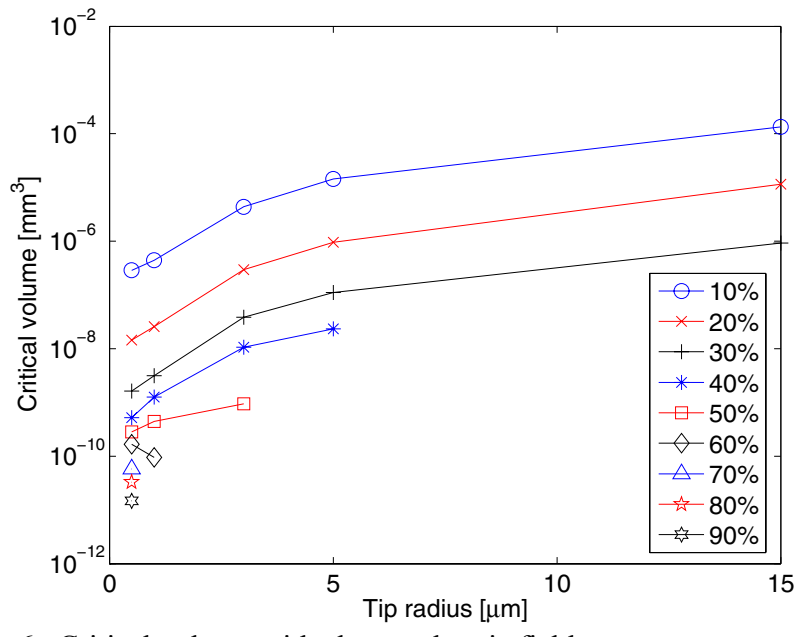

Fig.6. Critical volume with chosen electric fields.

To confirm our hypothesis, the cases in [4] are also analyzed with the electric fields from the previous analysis. In this analysis, gap spacing is varied from $0.7 \mathrm{~mm}$ to $20 \mathrm{~mm}$. The voltages with the same electric field in the cases of [4] are analyzed, and compared to the measured data and the analytic ones [11], which have an assumption of infinite plate. This analysis accounted for both the finite length of the plate electrode and the steel tube, which cannot be accomplished analytically. The tip radius is $0.9 \mu \mathrm{m}$ [4]. The field strength used is $9.8 \mathrm{MV} / \mathrm{cm}$ as in [4]. Fig. 7 shows that this analysis is well matched to the measured data.

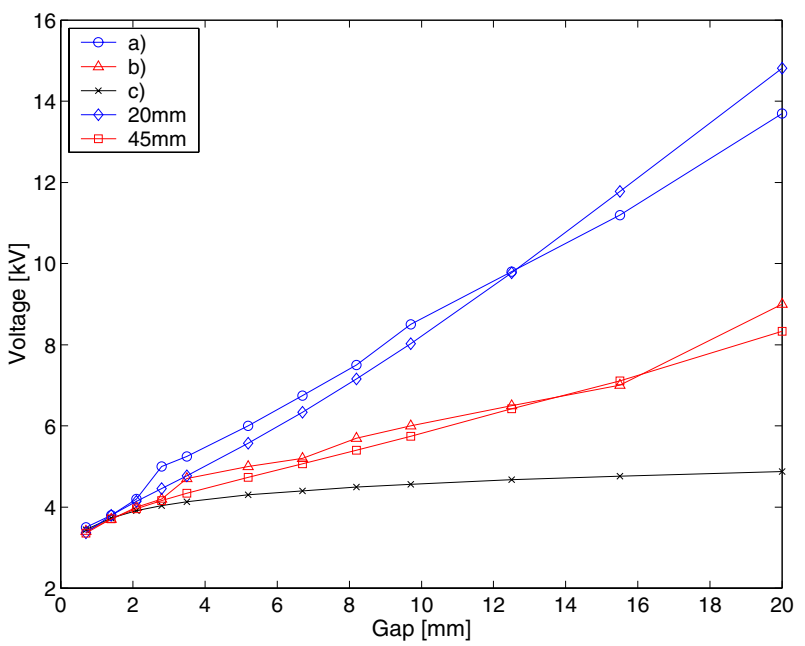

Fig.7. Initiation voltage with an initiation electric field of 9.8 $\mathrm{MV} / \mathrm{cm}$. a) Measurement of $20 \mathrm{~mm}$ plate case, b) measurement of 45 $\mathrm{mm}$ plate case, (c) theoretical curve from [11], and the analysis results. a) and b) are from [4].

To assess the sensitivity of the match, $9.62 \mathrm{MV} / \mathrm{cm}(40 \%)$, $10.83 \mathrm{MV} / \mathrm{cm}(45 \%)$ and $12.03 \mathrm{MV} / \mathrm{cm}(50 \%)$ were analyzed. Fig. 8 and Fig. 9 show the results of the $20 \mathrm{~mm}$ and $45 \mathrm{~mm}$ cathodes, respectively. In the $20 \mathrm{~mm}$ case, the measurement data from $0.7 \mathrm{~mm}$ to $12 \mathrm{~mm}$ gaps are between $40 \%$ and $45 \%$, and the measurement is lower than $40 \%$ at gaps of $15 \mathrm{~mm}$ and 
$20 \mathrm{~mm}$. In the $45 \mathrm{~mm}$ cathode case, the measured data falls between the calculated electric field distributions for field strengths that are at $40 \%$ and $45 \%$ of the maximum field. According to these results, the electric field needed to initiate streamers appears to be between $9.62 \mathrm{MV} / \mathrm{cm} \mathrm{(40 \% )}$ and $10.83 \mathrm{MV} / \mathrm{cm}(45 \%)$.

Both positive streamer cases from [3] and [4] have a same range of electric field for incipient streamers. The range is between $9.62 \mathrm{MV} / \mathrm{cm}$ and $12.03 \mathrm{MV} / \mathrm{cm}$.

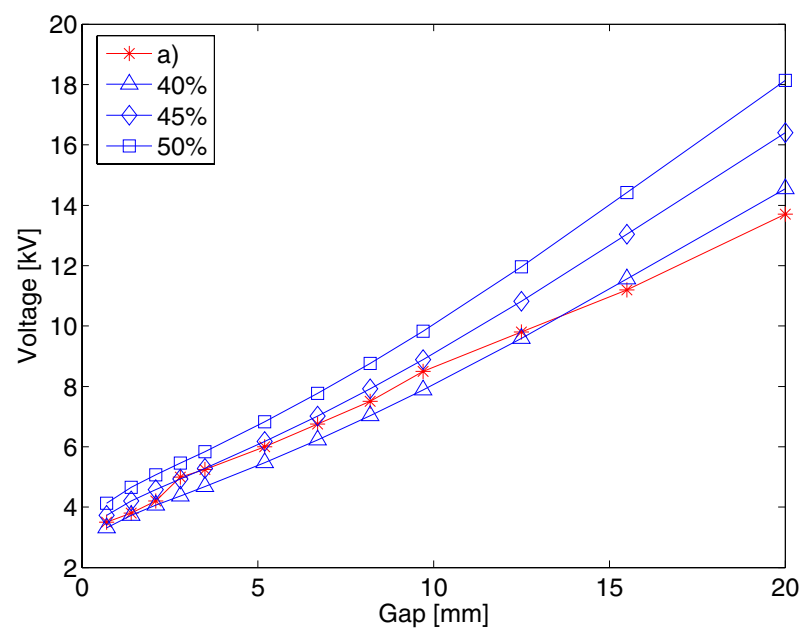

Fig.8. Comparison of calculations and data for $20 \mathrm{~mm}$ cathode with a) being the measurement from [4].

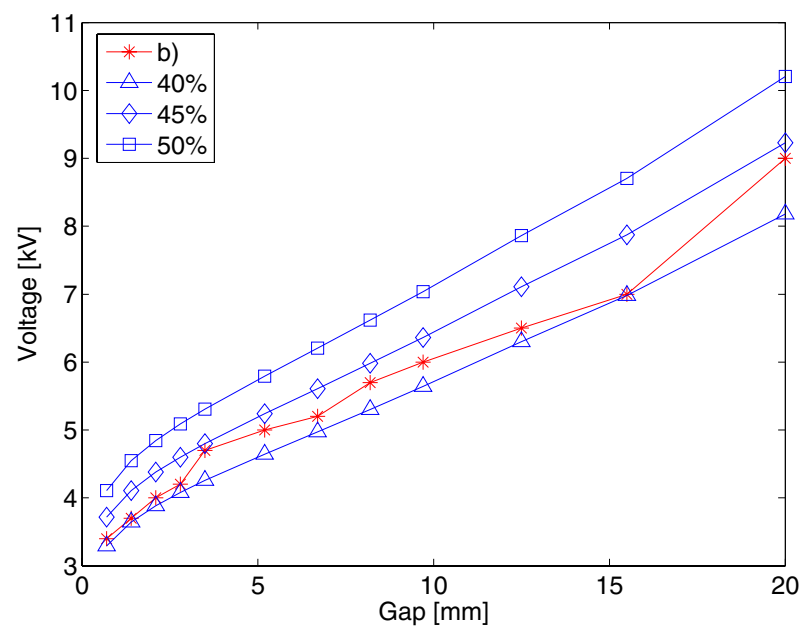

Fig.9. Comparison of calculations and data for $45 \mathrm{~mm}$ cathode with b) being the measurement from [4].

\section{CONCLUSION}

It is generally presumed that the initiation of streamers requires a specific level of electric field in the fluid, whose magnitude depends on the characteristics of the fluid. To test this presumption, the electric fields of previous works $[3,4]$ using different geometries were analyzed. The analysis suggests there is an electric field in the range between 9.62
$\mathrm{MV} / \mathrm{cm}$ and $12.03 \mathrm{MV} / \mathrm{cm}$ that yields consistency between the previous works.

\section{ACKNOWLEDGMENT}

The authors thank the Office of Naval Research for support of this work. In addition, they thank K. Davey for introducing them to software well suited to the solution of this class of problem.

\section{REFERENCES}

[1] A. Beroual, M. Zahn, A. Badent, K. Kist, A. J. Schwabe, H. Yamashita, K. Yamazawa, M. Danikas, W. G. Chadband, and Y. Torshin, "Propagation and structure of streamers in liquid dielectrics," IEEE Electr. Insul. Mag., vol. 14, no. 2, pp. 6-17, 1998.

[2] E. O. Forster, H. Yamashita, C. Mazzetti, M. Pompili, L. Caroli, and S. Patrissi, "The effect of the electrode gap on breakdown in liquid dielectrics," IEEE Trans. Dielect. Elect. Insulation, vol. 1, no. 3, pp. 440-446, June 1994.

[3] H. Yamashita, K. Yamazawa, and Y. S. Wang, "The effect of tip curvature on the prebreakdown streamer structure in cyclohexane," IEEE Trans. Dielect. Elect. Insulation, vol. 5, no. 3, pp. 396-401, June 1998.

[4] P. Gournay and O. Lesaint, "A study of the inception of positive streamers in cyclohexane and pentane," J. Phys. D: Appl. Phys., vol. 26, pp. 1966-1974, 1993.

[5] R. E. Hebner, "Measurements of electrical breakdown in liquids," in The Liquid State and its Electrical Properties, Plenum Press, New York, pp. 519-537, 1988.

[6] S. K. Venkatesh and M. S. Naidu, "Experimental and theoretical investigations on the statistics of time lags to corona inception and breakdown of SF6 in non-uniform electric fields," in Gaseous Dielectrics, vol. VIII, Christophorous and Olthoff, Eds. New York: Plenum, 1998, pp. 115-160.

[7] A. L. Kupershtokh and D. I. Karpov, "Stochastic featuers of liquid dielectric breakdown at small area of positive electrode," in Proc. $13^{\text {th }}$ International Conference on Dielectric Liquids, 1999, pp. 203-206.

[8] A. P. Ershov, and A. L. Kupershtokh, "Fluctuation model of liquid dielectrics breakdown with incomplete charge relaxation," in Proc. $11^{\text {th }}$ International Conference on Dielectric Liquids, 1993, pp. 194-198.

[9] E. F. Kelley and R. E. Hebner, "The electric field distribution associated with prebreakdown phenomena in nitrobenzene," $J$. Appl. Phys., vol. 52, pp. 191-195, 1981.

[10] H. A. Fowler, J. E. Devaney, and J. G. Hagedorn, "Shaping of filamentary streamers by the ambient field," in Proc. CEIDP, 1999, pp. 132-136.

[11] R. Coelho and J. Debeau, "Properties of the tip-plane configuration," J. Phys. D: Appl. Phys., vol. 4, pp. 1266-1280, 1971. 